\title{
Elevated anthranilic acid plasma concentrations in type 1 but not type 2 diabetes mellitus
}

\author{
Gregory Oxenkrug*1, Marieke van der Hart ${ }^{2}$ and Paul Summergrad ${ }^{1}$ \\ ${ }^{1}$ Psychiatry and Inflammation Program, Department of Psychiatry, Tufts University School of Medicine/Tufts Medical Center, Boston, MA, 02111, USA \\ ${ }^{2}$ Brains On-Line LLC, CA, USA
}

\begin{abstract}
Experimental data suggested involvement of tryptophan (Trp) - kynurenine (Kyn) pathway (TKP) in mechanisms of autoimmune, type 1 (T1D), and metabolic, type 2 (T2D), diabetes. However, clinical evaluations of TKP metabolites were limited to T2D. We assessed Trp, Kyn and TKP metabolites: anthranilic (AA), kynurenic (KYNA) and xanthurenic (XA) acids, in plasma samples of fifteen T1D, thirty T2D patients and twenty eight non-diabetic subjects by HPLC-mass spectrometry. Trp concentrations were higher in T1D than in T2D and controls while Kyn concentrations were not changed suggesting down-regulation of indoleamine-2,3-dioxygenase (IDO), a rate-limiting enzyme of TKP, in T1D. AA concentrations were 2.3-fold higher in T1D than in T2D and in controls. KYNA and XA concentrations were higher in T1D than in controls, and in previously reported T2D. AA elevation might be a specific feature of T1D. TKP shift towards AA formation in T1D may result from riboflavin deficiency, that increases AA in rats and baboons, and is highly associated with T1D but not T2D. AA augments autoimmune-induced apoptosis of pancreatic cells (PC) by increasing formation of antibodies to PC auto-antigen. Marked increase of AA was reported in rheumatoid arthritis, another autoimmune disorder. Trp, an essential amino acid for humans, is synthesized from AA by diabetogenic intestinal microbiome. AA down-regulates IDO by inhibition of Trp entry into cells. Resulting elevation of Trp attenuates Trp depletion-induced protection of PC against autoimmunity. Further studies of TKP might offer new tools for prevention and treatment of T1D and other autoimmune disorders.
\end{abstract}

\section{Introduction}

About 40 millions worldwide are diagnosed with type 1 diabetes (T1D), an organ specific autoimmune disease and one of the leading causes of disability and mortality [1]. Type 2 diabetes (T2D) is metabolic disorder that afflicts more than 344 million people worldwide and the 8th leading cause of death in the world [1]. Both T1D and T2D are highly associated with obesity [2]. Search of new strategies for prevention and treatment of diabetes requires further exploration of aetiopathogenetic mechanisms of diabetes development. Dysregulation of tryptophan (Trp) - kynurenine (Kyn) metabolic pathway (TKP) was suggested to contribute to development of T1D [3], T2D [4] and obesity $[5,6]$. Apart from protein metabolic pathway, $1-3 \%$ of $\operatorname{Trp}$ is converted into methoxyindoles (e.g., serotonin, $\mathrm{N}$-acetylserotonin, melatonin) while more than $95 \%$ of Trp is metabolized along TKP, a major source of $\mathrm{NAD}^{+}$in mammals [7]. Inflammation-induced indoleamine 2,3-dioxygenase-1 (IDO) or stress-induced tryptophan 2,3-dioxygenase-2 (TDO) activate Trp conversion into Kyn, a precursor of anthranilic (AA), kynurenic (KYNA), and (via 3-hydroxykynurenine, 3-HK) xanthurenic (XA) acids [7] (Figure 1). Elevated AA was found to predict the development of gestational diabetes [8] but not T2D [9]. However, we are not aware of clinical assessments of AA in T1D - the major aim of the present study.

\section{Materials and methods}

\section{Patients}

Overnight fasting plasma samples were collected from 15 (4 females and 11 males) T1D patients: four African Americans, six American Caucasians, and five Hispanics (age range from 33 to 69 years). T1D was diagnosed according to criteria of American Diabetes
Association. T1D patients received insulin treatment. Additionally, AA concentrations were evaluated in plasma samples of thirty T2D patients and twenty four non-diabetic (control) subjects described elsewhere [10]. Study was approved by Tufts Medical Center IRB,

\section{Assessment of TKP metabolites}

Plasma samples were stored at $-50^{\circ} \mathrm{C}$ until analysis. AA, Trp, Kyn, KYNA and XA plasma concentrations were analyzed by HPLC-mass spectrometry method.

Statistical Analysis. Results are presented as mean \pm standard error (Trp and Kyn in $\mu \mathrm{M}$ and AA, KYNA and XA in nM). Statistical significance was assessed as indicated in text.

\section{Results}

AA concentrations markedly (2.3fold) increased in T1D in comparison with controls. AA increase was robust: only two controls had AA values higher than mean value of T1D (Figure 2). AA concentrations in $\mathrm{T} 2 \mathrm{D}(83.57 \pm 16.5 \mathrm{nM})$ did not differ from controls (70.5 \pm 17.9$)$, and, consequently, were almost two times lower than in T1D $(165.8 \pm 21.9 \mathrm{nM})$ (Figure 2). Trp plasma concentrations were

Correspondence to: Gregory F. Oxenkrug, Tufts Medical Center, 800 Washington St, Box \#1007, Boston, MA, 02111, USA, E-mail: goxenkrug@tuftsmedicalcenter.org

Key words: type 1 diabetes, type 2 diabetes, anthranilic acid, kynurenine, tryptophan

Received: September 10, 2015; Accepted: September 22, 2015; Published: September 25, 2015 


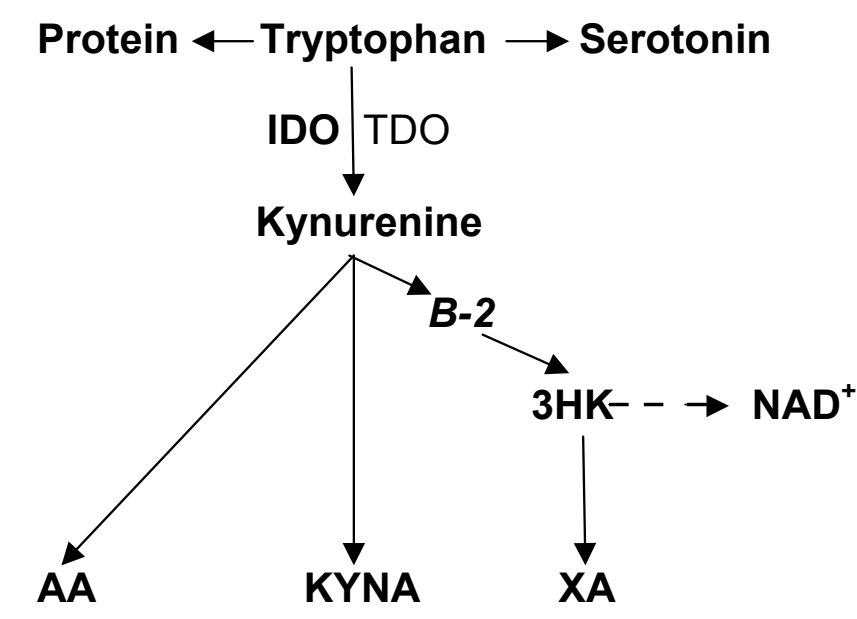

Abbreviations: TRP: tryptophan; KYN: kynurenine; AA: anthranilic acid; B-2: riboflavin 3-HK: 3hydroxykynurenine; KYNA: kynurenic acid; XA: xanthurenic acid; IDO indoleamine 2,3-dioxygenase; TDO: tryptophan 2,3-dioxygenase; $\mathrm{NAD}^{+}$: nicotinamide adenine dinucleotide

Figure 1. Tryptophan - Kynurenine pathway.

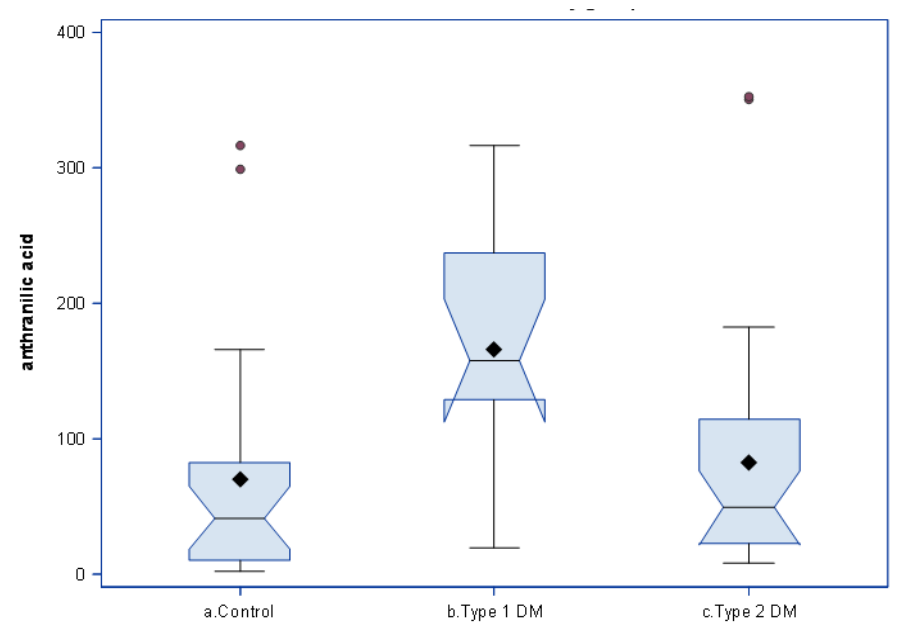

Figure 2. Plasma anthranilic acid concentrations in type 1 and type 2 diabetes.

Horizontal line shows median, diamond shows mean span of boxes is from 25th to 75th percentile. T1D in comparison with controls, $\mathrm{p}=0.005$. T1D in comparison with T2D, $\mathrm{p}=0.01$ (1-way ANOVA with Bonferroni correction)

elevated in T1D by $70 \%$ in comparison with controls (Table 1). Kyn plasma concentrations in T1D were not different from controls. Kyn:Trp ratio, a clinical index of IDO and TDO activity [11], was decreased by $40 \%$ in T1D patients. KYNA and XA concentrations were higher in T1D than in controls (Table 1).

\section{Discussion}

Major finding of the present study is a marked and robust increase of plasma AA concentrations in T1D in comparison with both nondiabetic (controls) and T2D subjects. There were no differences in AA plasma concentrations between $\mathrm{T} 2 \mathrm{D}$ and controls. Our results are in agreement with literature data of no differences between saliva AA concentrations of T2D patients and controls [12] and between plasma AA concentrations in cardiovascular patients with- and with-out T2D [13]. Additionally, elevated plasma AA concentrations did not predict development of T2D [9]. Therefore, one might suggest that increased
Table 1. Anthranilic acid and TKP metabolites in T1D.

\begin{tabular}{|l|c|c|c|}
\hline & $\begin{array}{c}\text { CONTROL } \\
(\mathbf{n = 2 4 )}\end{array}$ & $\begin{array}{c}\text { T1D } \\
(\mathbf{n = 1 4})\end{array}$ & P* \\
\hline $\mathrm{AA}(\mathrm{nM})$ & $70.5 \pm 17.9 \#$ & $165.8 \pm 21.9$ & 0.002 \\
\hline Kyn $(\mu \mathrm{M})$ & $1.77 \pm 0.09$ & $1.83 \pm 0.15$ & 0.45 \\
\hline Kyn:Trp & $2.50 \pm 0.13$ & $1.51 \pm 0.24$ & 0.04 \\
\hline Trp $(\mu \mathrm{M})$ & $70.8 \pm 2.5$ & $121.9 \pm 21.6$ & 0.0001 \\
\hline XA $(\mathrm{nM})$ & $11.7 \pm 1.2$ & $18.07 \pm 2.2$ & 0.01 \\
\hline KYNA(nM) & $35.4 \pm 2.7$ & $71.5 \pm 4.42$ & 0.0001 \\
\hline
\end{tabular}

\#; mean \pm standard error, *; Mann-Whitney, two-tailed test

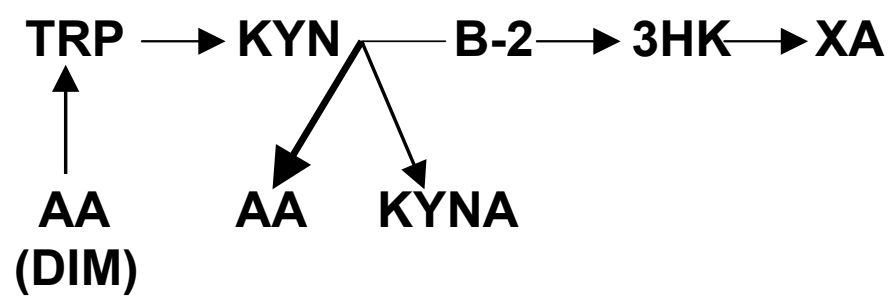

Abbreviations: TRP: tryptophan; KYN: kynurenine; AA: anthranilic acid; B-2: riboflavin; 3-HK: 3hydroxykynurenine; KYNA: kynurenic acid; XA: xanthurenic acid; DIM: diabetogenic intestinal microbiome

Figure 3. Hypothetical changes of Tryptophan-Kynurenine metabolism in T1D.

plasma AA concentration is a specific feature of T1D.

AA is rarely measured in human studies. Increased levels of AA were found in the synovial fluid of rheumatoid arthritis, an autoimmune disease [14], and in osteoporosis [15]. Osteoporosis is highly associated with T1D but not T2D [16]. Therefore, elevation of AA in osteoporosis may depend on association of osteoporosis with T1D.

Vitamin B-2 (riboflavin) deficiency might be one of the causes of increased AA formation. Excretion of large amount of AA was described in riboflavin deficient rats [17] and baboons [18]. AA, KYNA and 3-HK are compete for Kyn as a substrate for their biosynthesis (Figures 1 and 2). Conversion of Kyn into AA and KYNA is catalyzed by enzymes utilizing vitamin B6 as a co-factor [7] while riboflavin is a co-factor for enzymes catalyzing formation of 3-HK [5]. Therefore, riboflavin deficiency increases availability of Kyn as a substrate for biosynthesis of AA at the expense of 3-HK formation (Figure 3). Indeed, riboflavin-deficient baboons showed not only twelvefold increase in the AA excretion, but a tenfold decrease in 3-HK [18] suggesting a shift of Kyn metabolism from formation of 3-HK to production of AA (Figure 3). Clinical studies found fourfold greater percentage of riboflavin deficiency in T1D children (6 - 16 years of age) than in non diabetics [19]. Deterioration of riboflavin metabolism was associated with indices of glycemia and glucosuria in T1D children (9-15 years of age) and adults, but not in T2D and healthy persons [20].

The relevance of increased AA to mechanisms of T1D development might be stressed by observation of AA-induced exacerbation of arthritis lesions in adjuvant-induced model of autoimmune arthritis in rats [21]. Most importantly, AA augments production of antibodies to sheep erythrocytes in mice [21]. Assuming that AA might similarly augment production of antibodies to pancreatic cell (PC) auto-antigen, one might suggest that elevated plasma AA potentiates antibody attack leading to autoimmune-induced apoptosis of PC in T1D.

Alternatively, elevated plasma AA concentrations might reflect 
adaptive (protective) response to (against) T1D. Thus, AA, in contrast to 3-hydroxyanthranilic acid, exerts anti-oxidant effects in iron autooxidation assay [22]. Additionally, AA was found to inhibit enzyme catalyzing metabolism of 3-hydroxyantranilic acid into quinolinic acid, the most toxic TKP derivative [23].

Present study revealed increased plasma concentrations of Trp in $\mathrm{T} 1 \mathrm{D}$, that, in association with unchanged (in comparison with controls) Kyn concentrations, resulted in decreased Kyn:Trp ratio. Observed decreased of Kyn:Trp ratio is unlikely caused by insulin treatment since insulin activates Trp conversion into Kyn in rats [24]. Kyn:Trp ratio in T2D did not differ from controls [8,9]. Decreased Kyn:Trp ratio suggests down-regulation of IDO, and, consequently, elevated Trp. Trp availability was proposed to regulate immune responses, i.e., IDO-induced depletion of Trp protected PC from destruction by autoimmune processes [3] while inhibition of IDO exacerbated STZ-induced diabetes in C57BL/6 mice. However, IDO was poorly expressed and its activity was not inducible in 4 months old non-obese diabetic (NOD) mice [25]. Present results of increased Trp and decreased Kyn:Trp ratio in T1D are the first (to our knowledge) clinical observation of impairment of IDO-dependent protection of PC against autoimmune destruction in T1D.

Elevation of plasma Trp concentration, besides down-regulation of Trp conversion into Kyn, might be caused by increased biosynthesis of Trp. Trp is essential amino acid for humans but it could be synthesized from AA by bacteria [26]. Composition of the intestinal microbiome influences the development of T1D [27]. NOD mice were reported to harbor intestinal microbes that induce diabetes [28].

Increased availability of Trp produced from AA by intestinal microbiome might eventually down-regulate IDO by inhibiting Trpselective transport system that facilitates the entry of Trp into cells and optimize the substrate availability for IDO [29].

Increased plasma concentrations of two other studied TKP derivatives, KYNA and XA, are unlikely specific for T1D considering that increased concentrations of XA and KYNA (although less prominent) were observed previously in T2D as well [10]. XA was reported to induce pathological apoptosis of PC through caspase 3-dependent mechanism [30].

Increased hypothalamic KYNA content was reported in STZinduced diabetes [31]. It is noteworthy that KYNA, but not other kynurenines, selectively activates GPR35 receptor on the surface of immune cells and inhibits synthesis of IDO-inducing proinflammatory cytokines. KYNA, in low concentrations, was shown to stimulate proliferation of T-cells [32].

In conclusion, elevation of AA formation might be a specific feature of T1D (and other autoimmune disorders). We suggest that down-stream Kyn metabolism is shifted toward AA production by riboflavin deficiency. AA augments autoimmune-induced apoptosis by potentiating production of antibodies to $\mathrm{PC}$ auto-antigen. Increased formation of Trp from AA by diabetogenic intestinal microbiome down-regulates IDO via inhibition of Trp entry into cell. Downregulation of IDO attenuates Trp depletion-induced protection of PC against autoimmunity in T1D (Figure 3).

Further studies of AA involvement in aetiopathogenesis of T1D might offer new tools for prevention and treatment of T1D and other autoimmune organ-specific disorders.

\section{Acknowledgments}

GF Oxenkrug is a recipient of NIMH104810 grant. We appreciate Bioreclamation IVT, NY, USA, for help in collection of plasma samples.

\section{References}

1. Tao Z, Shi A, Zhao J (2015) Epidemiological Perspectives of Diabetes. Cell Biochem Biophys 73: 181-185. [Crossref]

2. Minges KE, Whittemore R, Grey M (2013) Overweight and obesity in youth with type 1 diabetes. Figure Annu Rev Nurs Res 31: 47-69. [Crossref]

3. Grohmann U, Fallarino F, Bianchi R, Orabona C, Vacca C, et al. (2003) A defect in tryptophan catabolism impairs tolerance in nonobese diabetic mice. Figure J Exp Med 198: 153-160. [Crossref]

4. Oxenkrug G (2013) Insulin Resistance and Dysregulation of Tryptophan-Kynurenine and Kynurenine Nicotinamide Adenine Dinucleotide Metabolic Pathways. Mol Neurobiol 48: 294-301. [Crossref]

5. Oxenkrug GF (2010) Metabolic syndrome, age-associated neuroendocrine disorders, and dysregulation of tryptophan-kynurenine metabolism. Figure Ann N Y Acad Sci 1199: 1-14. [Crossref]

6. Wolowczuk, Hennart B, Leloire A, Bessede A, Soichot M, et al (2012) Tryptophan metabolism activation by indoleamine 2,3-dioxygenase in adipose tissue of obese women: an attempt to maintain immune homeostasis and vascular tone. Am J Physiol Regul Integr Comp Physiol. 303:R135-43. [Crossref]

7. Schwarcz R, Bruno JP, Muchowski PJ, Wu HQ (2012) Kynurenines in the mammalian brain: when physiology meets pathology. Figure Nat Rev Neurosci 13: 465-477. [Crossref]

8. Bentley-Lewis R, Huynh J, Xiong G, Lee H, Wenger J, et al. (2015) Metabolomic profiling in the prediction of gestational diabetes mellitus. Figure Diabetologia 58: 1329-1332. [Crossref]

9. Wang TJ, Larson MG, Vasan RS, Cheng S, Rhee EP, et al. (2011) Metabolite profiles and the risk of developing diabetes. Figure Nat Med 17: 448-453. [Crossref]

10. Oxenkrug GF (2015) Increased Plasma Levels of Xanthurenic and Kynurenic Acids in Type 2 Diabetes. Figure Mol Neurobiol 52: 805-810. [Crossref]

11. Schröcksnadel K, Widner B, Bergant A, Neurauter G, Schröcksnadel H, et al. (2003) Tryptophan degradation during and after gestation. Figure Adv Exp Med Biol 527: 77-83. [Crossref]

12. Bosco P, Stokowska W, Gorska M, Kusharewicz I, Pawlak D, et al. (2006) Tryptophan metabolites via kynurenine pathway in saliva of diabetic patients. Dent Med Probl 41 $21-25$.

13. Pedersen ER, Tuseth N, Eussen SJ, Ueland PM, Strand E, et al. (2015) Association of plasma kynurenines with risk of acute myocardial infarction in patients with stable angina pectoris. Arterioscler Thromb Vasc Biol 35:455-62. [Crossref]

14. Igari T, Tsuchizawa M, Shimamura T (1987) Alteration of tryptophan metabolism in the synovial fluid of patients with rheumatoid arthritis and osteoarthritis. Figure Tohoku J Exp Med 153: 79-86. [Crossref]

15. Forrest CM, Mackay GM, Oxford L, Stoy N, Stone TW, et al. (2006) Kynurenine pathway metabolism in patients with osteoporosis after 2 years of drug treatment. Figure Clin Exp Pharmacol Physiol 33: 1078-1087. [Crossref]

16. Chau DL, MD and Steven V. Edelman, MD (2002) Osteoporosis and Diabetes. Clinical Diabetes 20:153-157.

17. Charconnet-Harding F, Dalgliesh CE, Neuberger A (1953) The relation between riboflavin and tryptophan metabolism, studied in the rat. Figure Biochem J 53: 513521. [Crossref]

18. Verjee ZH (1975) Tryptophan metabolism in baboons: effect of riboflavin and pyridoxine deficiency. Figure Acta Vitaminol Enzymol 29: 198-201. [Crossref]

19. Cole HS, Lopez R, Cooperman JM (1976) Riboflavin deficiency in children with diabetes mellitus. Figure Acta Diabetol Lat 13: 25-29. [Crossref]

20. Kodentsova VM, Vrzhesinskaia OA, Sokol'nikov AA, Alekseeva IA, Spirichev VB (1993) [Metabolism of riboflavin and B group vitamins functionally bound to it in insulin-dependent diabetes mellitus]. Vopr Med Khim 39(5): 33-6. [Crossref]

21. Kunitomo M, Tanaka Y, Yamada K, Yamaguchi Y, Bandô Y (1989) Enhancing activity of anthranilic acid on adjuvant arthritis in rats and antibody formation in mice. Figure Jpn J Pharmacol 50: 507-510. [Crossref] 
22. Chobot V, Hadacek F, Weckwerth W, Kubicova L (2015) Iron chelation and redox chemistry of anthranilic acid and 3-hydroxyanthranilic acid: A comparison of two structurally related kynurenine pathway metabolites to obtain improved insights into their potential role in neurological disease development. J Organomet Chem. 782:103110. [Crossref]

23. Guillemin GJ, Cullen KM, Lim CK, Smythe GA, Garner B, et al. (2007) Characterization of the kynurenine pathway in human neurons. Figure J Neurosci 27: 12884-12892. [Crossref]

24. Schor JM, Frieden E (1958) Induction of tryptophan peroxidase of rat liver by insulin and alloxan. Figure J Biol Chem 233: 612-618. [Crossref]

25. Fallarino F, Volpi C, Zelante T, Vacca C, Calvitti M, et al. (2009) IDO mediates TLR9driven protection from experimental autoimmune diabetes. Figure J Immunol 183: 6303-6312. [Crossref]

26. RYDON HN (1948) Anthranilic acid as an intermediate in the biosynthesis of tryptophan by Bact. typhosum. Figure Br J Exp Pathol 29: 48-57. [Crossref]

27. Peng J, Narasimhan S, Marchesi JR, Benson A, Wong FS, et al. (2014) Long term effect of gut microbiota transfer on diabetes development. Figure J Autoimmun 53: 85-94. [Crossref]
28. Brown K, Godovannyi A, Ma C, Zhang Y, Ahmadi-Vand Z, et al.(2015) Prolonged antibiotic treatment induces a diabetogenic intestinal microbiome that accelerates diabetes in NOD mice. ISME J: 1 -12

29. Bhutia YD, Babu E, Ganapathy V (2015) Interferon-? induces a tryptophan-selective amino acid transporter in human colonic epithelial cells and mouse dendritic cells. Biochim Biophys Acta 1848:453-62. [Crossref]

30. Malina HZ, Richter C, Mehl M, Hess OM (2001) Pathological apoptosis by xanthurenic acid, a tryptophan metabolite: activation of cell caspases but not cytoskeleton breakdown. Figure BMC Physiol 1: 7. [Crossref]

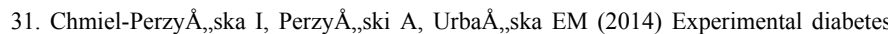
mellitus type 1 increases hippocampal content of kynurenic acid in rats. Figure Pharmacol Rep 66: 1134-1139. [Crossref]

32. MaÅ,aczewska J, Siwicki AK, WÃ $\tilde{3}^{3}$ jik RM, Kaczorek E, Turski WA (2014) Effect of oral administration of kynurenic acid on the activity of the peripheral blood leukocytes in mice. Figure Cent Eur J Immunol 39: 6-13. [Crossref]

33. Straub RH, Rokitzki L, Schumacher T, Hillmann C, Palitzsch KD, et al. (1993) [Patients with type-II diabetes mellitus and neuropathy have no deficiency of vitamins A, E, beta-carotene, B1, B2, B6, B12 and folic acid]. Figure Med Klin (Munich) 88 453-457. [Crossref]

Copyright: (C2015 Oxenkrug G. This is an open-access article distributed under the terms of the Creative Commons Attribution License, which permits unrestricted use, distribution, and reproduction in any medium, provided the original author and source are credited. 